Pacific Journal of Mathematic 


\section{A DYNAMICAL CRITERION FOR CONJUGATE POINTS}

\section{KurT KreITH}

This paper presents a technique for establishing the existence of conjugate points for real fourth order differential equations defined on an interval $[\alpha, \infty)$. The point $\beta>\alpha$ is conjugate to $\alpha$ if there exists a nontrivial solution $y(x)$ of the equation which satisfies

$$
y(\alpha)=y^{\prime}(\alpha)=0=y(\beta)=y^{\prime}(\beta) .
$$

An important feature of this technique is that it is not limited to equations of selfadjoint type and that the general theory applies to nonlinear equations as well.

For the special equation

$$
l[y] \equiv\left(p_{2}(t) y^{\prime \prime}\right)^{\prime \prime}+p_{0}(t) y=0 \quad\left(p_{2}(t)>0\right)
$$

criteria for the existence of conjugate points have been established by Leighton and Nehari [4] under the additional assumption $p_{0}(t)<$ 0 . Subsequent studies (see [6]) have extended parts of this theory to the general real selfadjoint equation

$$
l[y] \equiv\left(p_{2}(t) y^{\prime \prime}\right)^{\prime \prime}-\left(p_{1}(t) y^{\prime}\right)^{\prime}+p_{0}(t) y=0
$$

or the general real equation

$$
l[y] \equiv\left(p_{2}(t) y^{\prime \prime}-q_{2}(t) y^{\prime}\right)^{\prime \prime}-\left(p_{1}(t) y^{\prime}-q_{1}(t) y\right)^{\prime}+p_{0}(t) y=0 \text {. }
$$

replacing hypotheses on the coefficients with hypotheses specifying the nonexistence of solutions with certain orders of zeros. In this way, properties of solutions of (1.1), which were established in [4], became hypotheses which allowed the consideration of more general equations.

The present paper follows a similar pattern. In $\$ 2$, we consider a second order system which can be used to represent equations of the form (1.2) or (1.3) and allows a simple dynamical interpretation in terms of a particle of unit mass in a force field. By making a number of qualitative assumptions regarding this force field which are motivated by (1.1), we demonstrate the existence of conjugate points for such 
systems. In $\S 3$, we establish conditions on the coefficients of the differential system which assure that these qualitative assumptions are satisfied; these conditions on the coefficients of the system are translated to conditions on the coefficients of the related fourth order equation in $\$ 4$.

2. A related second order system. In this section we assume that the fourth order equation in question is represented by the second order system

$$
\begin{aligned}
& y^{\prime \prime}=a(t) y+b(t) x \\
& x^{\prime \prime}=c(t) y+d(t) x
\end{aligned}
$$

whose coefficients are continuous in $[\alpha, \infty)$. It has been shown by Whyburn [7] that the selfadjoint fourth order equation (1.2) can be represented in the form (2.1) with $b(t)=1 / p_{2}(t)>0$ and $a(t) \equiv$ $d(t)$. The author [1] has shown that the general real linear fourth order equation (1.3) can also be reduced to the form (2.1), with the nonselfadjointness reflected by the inequality of $a(t)$ and $d(t)$. In particular, the equation (1.1) can be represented in the form (2.1) with $b(t)=1 / p_{2}(t)$, $c(t)=-p_{0}(t)$, and $a(t)=d(t) \equiv 0$.

It will be helpful to interpret (2.1) as representing the motion of a particle of unit mass in the $(x, y)$-plane with $t$ denoting time. Our objective is to impose conditions on the force field

$$
\vec{F}(t)=\left(F_{x}(t), F_{y}(t)\right)=(c(t) y+d(t) x, a(t) y+b(t) x)
$$

which assure the existence of a conjugate point - i.e., the existence of a trajectory $C$ in the $(x, y)$-plane which is tangent to the $x$-axis at $t=\alpha$ and $t=\beta$.

This problem can be normalized by considering initial conditions of the form

$$
y(\alpha)=y^{\prime}(\alpha)=0 ; \quad x(\alpha)=1 ; \quad x^{\prime}(\alpha)=v_{0}
$$

Physically this corresponds to firing a particle of unit mass from $(x, y)=(1,0)$ tangent to the $x$-axis with velocity $v_{0}$ in the positive $x$ direction. The resulting one-parameter family of trajectories will be denoted by $C\left(v_{0}\right)$. We also denote by I, II, III, and IV the open quadrants of the $(x, y)$-plane.

Motivated by the system representation of (1.1), we consider the following conditions on the force field $\vec{F}$ : 
(A) If for some $t_{0} \geqq \alpha$ the quantities $y\left(t_{0}\right), y^{\prime}\left(t_{0}\right), x\left(t_{0}\right)$ and $x^{\prime}\left(t_{0}\right)$ are all nonnegative (but not all zero), then $y(t), y^{\prime}(t), x(t)$ and $\left.x^{\prime} t\right)$ are all positive for $t>t_{0}$.

(B) No trajectory $C\left(v_{0}\right)$ can remain in II for arbitrarily large values of $t$.

(C) No trajectory in I satisfies

(i) $x(t) \downarrow x_{0} \geqq 0$ and $y(t) \uparrow \infty$ as $t \rightarrow \infty$,

or

(ii) $y(t) \downarrow y_{0} \geqq 0$ and $x(t) \uparrow \infty$ as $t \rightarrow \infty$, nor can any trajectory in $I$ tend to a finite limit point $\left(x_{0}, y_{0}\right)$ in the closure of $I$ as $t \rightarrow \infty$.

(D) No trajectory can go directly from II to I to II.

Leмma 2.1. There exist values of $v_{0}$ such that $C\left(v_{0}\right)$ enters the closed lower half plane

$$
\{(x, y) \mid y \leqq 0\}
$$

Proof. By [1] the system (2.1) represents a fourth order linear differential equation of the form $l[y]=0$. If $\left\{y_{i}(t)\right\}(i=1, \cdots, 4)$ represents a fundamental set of solutions, one can find a linear combination $y(t)=\sum_{i=1}^{4} c_{i} y_{i}(t)$ having three preassigned zeros. In particular for any $\beta>\alpha$, there exists a solution $y(t)$ satisfying $y(\alpha)=y^{\prime}(\alpha)=y(\beta)=0$.

THEOREM 2.2. If conditions (A)-(D) are satisfied, then there exists a nontrivial solution $y(t), x(t)$ of (2.1) satisfying $y(\alpha)=y^{\prime}(\alpha)=$ $0=y(\beta)=y^{\prime}(\beta)$ for $\alpha<\beta<\infty$.

Proof. Solutions of (2.1) depend continuously on the initial data, and for the normalized problem under consideration the only initial parameter is $v_{0}$. It follows that in any compact interval $[\alpha, \gamma], C\left(v_{0}\right)$ can be approximated uniformly by trajectories of the form $C\left(v_{0}+\epsilon\right)$ for $|\epsilon|$ sufficiently small. Consider first

$$
V_{1}=\left\{v_{0} \mid C\left(c_{0}\right) \text { enters } I I I \cup I V\right\} .
$$

By Lemma 2.1 there are trajectories $C\left(v_{0}\right)$ which are either tangent to the $x$-axis or enter the lower half plane, and we may therefore assume that $V_{1}$ is not empty. Furthermore, (A) implies that if $v_{0} \geqq 0$, then $C\left(v_{0}\right)$ is "trapped" in I for all $t>\alpha$, so that $V_{1}$ is also bounded above by zero. Finally the continuous dependence of $C\left(v_{0}\right)$ on $v_{0}$ implies that $V_{1}$ is an open subset of $\mathbf{R}$. Consider next

$V_{2}=\left\{v_{0} \mid C\left(v_{0}\right)\right.$ remains in the open upper half plane for all $\left.t>\alpha\right\}$. 
By (A), $V_{2}$ contains $[0, \infty)$ and is therefore not empty. We shall show that $V_{2}$ is also open. Since by $(\mathrm{B}), C\left(v_{0}\right)$ cannot remain in II for all $t>\gamma$, and by (D) no trajectory can go from II to I to II, we may restrict our attention to trajectories $C\left(v_{0}\right)$ for which there exists $\gamma>\alpha$ such that $C\left(v_{0}\right)$ remains in $I$ for $t>\gamma$. Condition (C) rules out limit points in $I$ as well as asymptotic trajectories for which $x^{\prime}(t)$ and $y^{\prime}(t)$ have opposite signs for all $t>\gamma$, so that every trajectory which remains in $I$ eventually has positive values of $y(t), y^{\prime}(t), x(t)$, and $x^{\prime}(t)$. Because of the continuous dependence of $C\left(v_{0}\right)$ on the parameter $v_{0}$, it follows that neighboring trajectories will also eventually have positive values of $y(t), y^{\prime}(t), x(t)$, and $x^{\prime}(t)$ and that $V_{2}$ is an open subset of $\mathbf{R}$.

Consider now $\tilde{v}=\sup V_{1}$. Since $\tilde{v}$ belongs to neither $V_{1}$ nor $V_{2}$, it follows that $C(\tilde{v})$ lies in $\overline{I \cup I I}$ but not in $I \cup I I-$ i.e., $C(\tilde{v})$ must be tangent to the $x$-axis for some $x=\beta>\alpha$. This completes the proof.

3. Criteria for conjugate points. We now consider the task of imposing conditions on the system (2.1) such that properties (A) -(D) are satisfied. A basic assumption which will be made throughout is that the coefficients of $(2.1)$ are positive in $[\alpha, \infty)$. The reason for this assumption is the following.

THEOREM. 3.1. If the coefficients of (2.1) are positive in $[\alpha, \infty)$, then (A) is satisfied.

Proof. This follows readily from the integral representation

$$
\begin{aligned}
& y(t)=y\left(t_{0}\right)+y^{\prime}\left(t_{0}\right)\left(t-t_{0}\right)+\int_{t_{0}}^{t} \int_{t_{0}}^{s}[a(\tau) y(\tau)+b(\tau) x(\tau)] d \tau d s \\
& x(t)=x\left(t_{0}\right)+x^{\prime}\left(t_{0}\right)\left(t-t_{0}\right)+\int_{t_{0}}^{t} \int_{t_{0}}^{s}[c(\tau) y(\tau)+d(\tau) x(\tau)] d \tau d s
\end{aligned}
$$

for solutions of (2.1).

In order to establish (B), we introduce a vector representation

$$
Y^{\prime \prime}=A(t) Y
$$

for (2.1) where

$$
Y(t)=\left(\begin{array}{l}
x(t) \\
y(t)
\end{array}\right) ; \quad A(t)=\left(\begin{array}{ll}
d(t) & c(t) \\
b(t) & a(t)
\end{array}\right)
$$


Also $H$ will denote a constant vector

$$
H=\left(\begin{array}{r}
-1 \\
1
\end{array}\right)
$$

and inner products will be denoted by $\langle\cdot, \cdot\rangle$ so that

$$
\langle H, Y(t)\rangle=y(t)-x(t)
$$

etc.

THEOREM 3.2. If $c(t) \geqq a(t)>0$ and $b(t) \geqq d(t)>0$ in $[\alpha, \infty)$ and the equation

$$
u^{\prime \prime}+\min \{b(t)-d(t), c(t)-a(t)\} u=0
$$

is oscillatory at $t=\infty$, then (B) is satisfied.

Proof. If $Y$ is a nonzero element in $I I$ then $x<0, y>0$ and

$$
\langle H, Y\rangle=y-x>0
$$

Also

$$
-\langle H, A Y\rangle=(b-d)(-x)+(c-a) y \geqq \min \{b-d, c-a\}(y-x) \geqq 0 .
$$

Therefore

$$
-\frac{\langle H, A Y\rangle}{\langle H, Y\rangle} \geqq \min \{b-d, c-a\}
$$

for all $Y \in I I$.

Define $m(t)=\min \{b(t)-d(t), c(t)-a(t)\}$ and let $t_{1}<t_{2}<\cdots$ be the zeros of an oscillatory solution of (3.2) where $t_{k} \uparrow \infty$. If $Y(t)$ remains in II for all $t \geqq \gamma$, then $\langle H, Y(t)\rangle>0$ in $[\gamma, \infty)$ and a direct calculation yields

$$
\frac{d}{d t}\left[u u^{\prime}-u^{2} \frac{\left\langle H, Y^{\prime}\right\rangle}{\langle H, Y\rangle}\right]=-m u^{2}-u^{2} \frac{\langle H, A Y\rangle}{\langle H, Y\rangle}+\left[u^{\prime}-u \frac{\left\langle H, Y^{\prime}\right\rangle}{\langle H, Y\rangle}\right]^{2}
$$

If $t_{k+1}>t_{k} \geqq \gamma$ we have

$$
0=\left[u u^{\prime}-u^{2} \frac{\left\langle H, Y^{\prime}\right\rangle}{\langle H, Y\rangle}\right]_{t_{k}}^{t_{k+1}} \geqq-\int_{t_{k}}^{t_{k+1}}\left[m+\frac{\langle H, A Y\rangle}{\langle H, Y\rangle}\right] u^{2} d t
$$


with equality if and only if $u(t) \equiv\langle H, Y(t)\rangle$ in $\left(t_{k}, t_{k+1}\right)$. Since $\langle H, Y(t)\rangle$ is assumed positive in $[\gamma, \infty)$ the above inequality must be strict. But this contradicts (3.3) and completes the proof.

Condition $(C)$ requires that we preclude certain asymptotic paths and paths of finite length in $I$.

TheOREM 3.3. If

$$
\int^{\infty} t b(t) d t=\infty \text { and } \quad \int^{\infty} t c(t) d t=\infty
$$

then condition (C) is satisfied.

Proof. Consider first an asymptotic trajectory in $I$ for which $y(t) \downarrow y_{0} \geqq 0$ and $x(t) \uparrow \infty$ as $t \rightarrow \infty$. Since $x^{\prime \prime}>0$ in $I$ there exist positive constants $k$ and $\gamma$ such that $x(t) \geqq k t$ for $t \geqq \gamma$. Since $y^{\prime \prime} \geqq b(t) x(t)$ in $I$ we have

$$
y^{\prime}(t) \geqq y^{\prime}\left(t_{0}\right)+k \int_{t_{0}}^{t} t b(t) d t
$$

Thus the first part of (3.4) is inconsistent with such asymptotic trajectories, and the second part of (3.4) similarly precludes asymptotic trajectories for which $x(t) \downarrow x_{0} \geqq 0$ and $y(t) \uparrow \infty$ as $t \rightarrow \infty$.

To deal with paths of finite length which might terminate in $\bar{I}$, we note that $x^{\prime \prime}>0$ and $y^{\prime \prime}>0$ at every point of $\bar{I}$ except $(0,0)$. Thus the origin is the only equilibrium point in $\bar{I}$ and the only point at which finite paths might terminate.

There are two cases to consider in completing the proof:

(i) The trajectory never leaves $\bar{I}$. In this case $y^{\prime \prime}>0$ for all $t>\alpha$ and $y(t)$ is bounded away from zero in $[\gamma, \infty)$ for every $\gamma>\alpha$.

(ii) The trajectory leaves $\bar{I}$ and re-enters. In this case $x^{\prime}$ or $y^{\prime}$ is positive at the time the trajectory crosses into $I$ and the positivity of $x^{\prime \prime}$ and $y^{\prime \prime}$ precludes the possibility of the trajectory approaching the origin.

Finally we note that a very similar argument to that used above establishes (D). If a trajectory enters $I$ from $I I$ at time $t_{0}$, then $x^{\prime}\left(t_{0}\right)>0$ when $C\left(v_{0}\right)$ enters $I$. Since $x^{\prime \prime}>0$ in $I, x^{\prime}(t)$ is positive as long as $C\left(v_{0}\right)$ remains in $I$ and therefore $C\left(v_{0}\right)$ cannot return directly to $I I$ from $I$.

Collecting all the conditions imposed above on the coefficients of (2.1) we can state our principal result. 
THEOREM 3.4. If the coefficients of (2.1) satisfy

(i) $\quad c(t) \geqq a(t)>0$

(ii) $b(t) \geqq d(t)>0$

in $[\alpha, \infty)$ and

(iii) $u^{\prime \prime}+\min \{b(t)-d(t), c(t)-a(t)\} u=0$ is oscillatory at $t=\infty$,

(iv) $\int^{\infty} t b(t) d t=\int^{\infty} t c(t) d t=\infty$,

then there exists a nontrivial solution $y(t), x(t)$ of (2.1) satisfying $y(\alpha)=y^{\prime}(\alpha)=0=y(\beta)=y^{\prime}(\beta)$ for some $\beta>\alpha$.

4. Application to fourth order equations. In [1] the author shows how to reduce (1.3) to the form

$$
l[y] \equiv\left(p_{2}(t) y^{\prime \prime}\right)^{\prime \prime}-\left(p_{1}(t) y^{\prime}\right)^{\prime}+q_{1}(t) y^{\prime}+p_{0}(t) y=0
$$

and that there is a one-to-one correspondence between equations of the form (4.1) with $p_{2}(t)>0$ and systems of the form (2.1) with sufficiently regular coefficients and $b(t)>0$. This correspondence is obtained by defining

$$
x(t)=\frac{1}{b(t)} y^{\prime \prime}(t)-\frac{a(t)}{b(t)} y(t)
$$

and setting

$$
\begin{aligned}
& a=\frac{p_{1}-\int_{\alpha}^{t} q_{1}}{2 p_{2}} \\
& b=\frac{1}{p_{2}} \\
& c=\frac{p_{1}^{2}-\left(\int_{\alpha}^{t} q_{1}\right)^{2}}{4 p_{2}}-\frac{p_{1}^{\prime \prime}-q_{1}^{\prime}}{2}-p_{0} \\
& d=\frac{p_{1}+\int_{\alpha}^{t} q_{1}}{2 p_{2}} .
\end{aligned}
$$


These equations can be solved for $p_{2}, p_{1}, p_{0}$, and $q_{1}$ to yield

$$
\begin{aligned}
& p_{2}=\frac{1}{b} \\
& p_{1}=\frac{a+d}{b} \\
& q_{1}=\left(\frac{d-a}{b}\right)^{\prime} \\
& p_{0}=\frac{a d}{b}-\left(\frac{a}{b}\right)^{\prime}-c .
\end{aligned}
$$

This transformation makes it routine to apply Theorem 3.4 to the equation (4.1), though the transformation required to represent (1.3) in the form (4.1) makes the general application more involved.

It is of interest to examine the hypotheses of Theorem 3.4 for the special case $a(t) \equiv 0$ and $d(t) \equiv 0$ where our considerations reduce to the fourth order equation

$$
\left(p_{2}(t) y^{\prime \prime}\right)^{\prime \prime}+p_{0}(t) y=0 \quad\left(p_{0}(t)<0\right)
$$

considered by Leighton and Nehari in Part 1 of [4]. Condition (iii) of Theorem 3.4 is then satisfied if

$$
u^{\prime \prime}+\min \left\{\frac{1}{p_{2}(t)},-p_{0}(t)\right\} u=0
$$

is oscillatory at $\infty$. By the Sturm comparison theorem, (4.3) oscillatory implies that both $u^{\prime \prime}+\left(1 / p_{2}(t)\right) u=0$ and $u^{\prime \prime}-p_{0}(t) u=0$ are oscillatory at $\infty$, but one would not expect the converse to be true without some further hypotheses regarding the asymptotic behavior of $p_{2}(t)$ and $p_{0}(t)$. By a well known oscillation criterion of Kneser, (4.3) is oscillatory if

$$
\liminf _{t \rightarrow \infty}\left[t^{2} \min \left\{\frac{1}{p_{2}(t)},-p_{0}(t)\right\}\right]>\frac{1}{4}
$$

and this gives some measure of allowable rates of decay for $1 / p_{2}(t)$ and $p_{0}(t)$.

Condition (iv) of Theorem 3.4 becomes

$$
\int^{\infty} \frac{t}{p_{2}(t)} d t=-\int^{\infty} t p_{0}(t)=\infty
$$


While these conditions also put limits on the rate of decay of $1 / p_{2}(t)$ and $p_{0}(t)$, they are not sufficient to assure the oscillatory behavior of $u^{\prime \prime}+1 / p_{2}(t) u=0$ and $u^{\prime \prime}-p_{0}(t) u=0$. For example, $u^{\prime \prime}+1 / 4 t^{2}$ is nonoscillatory at $\infty$ but yet $\int^{\infty} 1 / 4 t d t=\infty$.

A special case of Theorem 6.2 of [4] assures the existence of a conjugate point for any $\alpha<\infty$ if

$$
\liminf _{t \rightarrow \infty} t^{2} \frac{1}{p_{2}(t)}>\frac{1}{4} \text { and } \quad \liminf _{t \rightarrow \infty} t^{2}\left(-p_{0}(t)\right)>\frac{1}{4}
$$

while it precludes the existence of conjugate points for sufficiently large $\alpha$ if

$$
\limsup _{t \rightarrow \infty} t^{2} \frac{1}{p_{2}(t)}<\frac{1}{4} \text { and } \quad \limsup _{t \rightarrow \infty} t^{2}\left(-p_{0}(t)\right)<\frac{1}{4}
$$

While (4.4) is slightly stronger than (4.5), the two conditions are roughly equivalent, and this comparison therefore suggests that the results of Theorem 3.4 are reasonably sharp even in this special case.

One is tempted to conjecture that the oscillatory behavior of both

$$
u^{\prime \prime}+\frac{1}{p_{2}(t)} u=0 \quad \text { and } \quad u^{\prime \prime}-p_{0}(t) u=0
$$

should insure the existence of conjugate points for (4.2) for all $\alpha<\infty$.

5. Concluding remarks. The techniques presented here are not quite as sensitive as those of [4] in the special case of equation (4.2). Their principal virtue is that they apply to non selfadjoint equations such as (4.1) and (1.3).

Several authors have used comparison theorems to establish lower bounds for conjugate points of nonselfadjoint equations of order $2 n$, and have thereby also established criteria for this disconjugacy (see for instance [5], [6], and [7]). However, I know of no results which establish upper bounds in the nonselfadjoint case if $n>1$.

Theorem 3.4 at least gives criteria for the existence of a conjugate point in the nonselfadjoint case. The question of how to obtain specific upper bounds for such conjugate points unfortunately remains unanswered, but it is hoped that these techniques may also prove useful in this connection. 


\section{REFERENCES}

1. K. Kreith, A nonselfadjoint dynamical system, Proc. Edinburgh Math. Soc., 19 (1974), 77-87.

2. - Disconjugacy criteria for nonselfadjoint differential equations of even order, Canad. J. Math., 23 (1971), 644-652.

3. - Oscillation properties of nonselfadjoint differential equations, Proceedings of the Conference on Difference on Differential Equations, Dundee, Scotland. Springer Verlag (1972), 294-297.

4. W. Leighton and Z. Nehari, On the oscillation of solutions of selfadjoint linear differential equations of fourth order, Trans. Amer. Math. Soc., 89 (1958), 325-377.

5. W. T. Reid, Oscillation criteria for linear differential systems with complex coefi cients, Pacific J. Math., 6 (1956), 733-751.

6. J. Ridenhour and T. Sherman, Conjugate points for fourth order linear differential equations, SIAM J. Appl. Math., 22 (1972), 599-603.

7. W. M. Whyburn, On selfadjoint ordinary differential equations of the fourth order, Amer. J. Math., 52 (1930), 171-196.

Received March 5, 1974.

University of California, Davis 


\section{CONTENTS}

Zvi Artstein and John A. Burns, Integration of compact set-valued functions

J. A. Beachy and W. D. Blair, Rings whose faithful left ideals are cofaithful

Mark Benard, Characters and Schur indices of the unitary reflection group $[321]^{3}$

H. L. Bentley and B. J. Taylor, Wallman rings ............................. 15

E. Berman, Matrix rings over polynomial identity rings II ............... 37

Simeon M. Berman, A new characterization of characteristic functions of absolutely continuous distributions ........................ 323

Monte B. Boisen, Jr. and Philip B. Sheldon, Pre-Prüfer rings ............ 331

A. K. Boyle and K. R. Goodearl, Rings over which certain modules are injective

J. L. Brenner, R. M. Crabwell and J. Riddell, Covering theorems for finite nonabelian simple groups. $V$

H. H. Brungs, Three questions on duo rings .................................... 345

Iracema M. Bund, Birnbaum-Orlicz spaces of functions on groups ....351

John D. Elwin and Donald R. Short, Branched immersions between 2-manifolds of higher topological type

J. K. Finch, The single valued extension property on a Banach space ................................................................................. 61

J. R. Fisher, A Goldie theorem for differentiably prime rings ............ 71

Eric M. Friedlander, Extension functions for rank 2, torsion free abelian groups ...................................................................... 371

J. Froemke and R. Quackenbusch, The spectrum of an equational class of groupoids

B. J. Gardner, Radicals of supplementary semilattice sums of

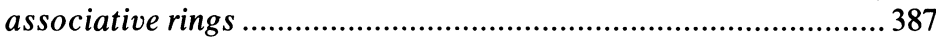

Shmuel Glasner, Relatively invariant measures ...............................393

G. R. Gordh, Jr. and Sibe Mardešić, Characterizing local connectedness in inverse limits...

S. Graf, On the existence of strong liftings in second countable topological spaces

S. Gudder and D. Strawther, Orthogonally additive and orthogonally increasing functions on vector spaces ........................................427

F. Hansen, On one-sided prime ideals .......................................... 79

D. J. Hartfiel and C. J. Maxson, A characterization of the maximal monoids and maximal groups in $\beta x$.

Robert E. Hartwig and S. Brent Morris, The universal flip matrix and the generalized faro-shuffle 


\section{Pacific Journal of Mathematics}

Vol. 58, No. 1

March, 1975

John Allen Beachy and William David Blair, Rings whose faithful left ideals are cofaithful .................................... 1

Herschel Lamar Bentley and Barbara June Taylor, Wallman rings ........ 15

Elizabeth Berman, Matrix rings over polynomial identity rings. II ...... 37

Ann K. Boyle and Kenneth R. Goodearl, Rings over which certain modules are injective ................................. 43

J. L. Brenner, Robert Myrl Cranwell and James Riddell, Covering theorems for finite nonabelian simple groups. $V \ldots \ldots \ldots \ldots \ldots \ldots \ldots \ldots \ldots$

James Kenneth Finch, The single valued extension property on a Banach

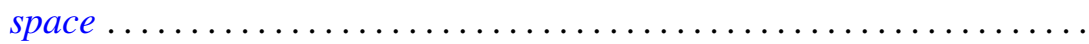

John Robert Fisher, A Goldie theorem for differentiably prime rings........ Friedhelm Hansen, On one-sided prime ideals .................... Jon Craig Helton, Product integrals and the solution of integral equations..........................................

Barry E. Johnson and James Patrick Williams, The range of a normal

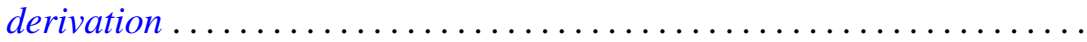

Kurt Kreith, A dynamical criterion for conjugate points ............ 123

Robert Allen McCoy, Baire spaces and hyperspaces .............. 133

John McDonald, Isometries of the disk algebra ................ 143

H. Minc, Doubly stochastic matrices with minimal permanents ......... 155

Shahbaz Noorvash, Covering the vertices of a graph by vertex-disjoint paths. ...

Theodore Windle Palmer, Jordan *-homomorphisms between reduced Banach*-algebras

Donald Steven Passman, On the semisimplicity of group rings of some

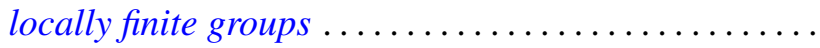

Mario Petrich, Varieties of orthodox bands of groups .

Robert Horace Redfield, The generalized interval topology on distributive

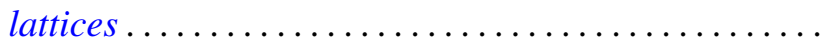

James Wilson Stepp, Algebraic maximal semilattices .... . .

Patrick Noble Stewart, A sheaf theoretic representation of rings with Boolean orthogonalities ........................

Ting-On To and Kai Wing Yip, A generalized Jensen's inequality......... 255

Arnold Lewis Villone, Second order differential operators with self-adjoint

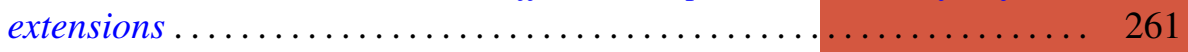

Martin E. Walter, On the structure of the Fourier-Stieltjes algebra ....... 267

John Wermer, Subharmonicity and hulls .................... 283

Edythe Parker Woodruff, A map of $E^{3}$ onto $E^{3}$ taking no disk onto a

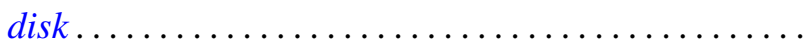

